\title{
The B Chromosomes of Prochilodus lineatus (Teleostei, Characiformes) Are Highly Enriched in Satellite DNAs
}

\author{
José Henrique Forte Stornioli ${ }^{1,+}+\mathbb{D}$, Caio Augusto Gomes Goes ${ }^{1,+} \mathbb{C}^{\infty}$, Rodrigo Milan Calegari ${ }^{1}{ }^{(}$, \\ Rodrigo Zeni dos Santos ${ }^{1}$, Leonardo Moura Giglio ${ }^{1}\left(\mathbb{D}\right.$, Fausto Foresti ${ }^{2}{ }^{(0)}$, Claudio Oliveira ${ }^{2}{ }^{(1)}$, \\ Manolo Penitente ${ }^{3}$, Fábio Porto-Foresti ${ }^{1}$ and Ricardo Utsunomia ${ }^{4, *(1)}$ \\ 1 Faculty of Sciences, São Paulo State University, Bauru, São Paulo 17033-360, Brazil; \\ jose.henrique@unesp.br (J.H.F.S.); caio.goes@unesp.br (C.A.G.G.); milan.calegari@unesp.br (R.M.C.); \\ rodrigo.zeni@unesp.br (R.Z.d.S.); leonardo.moura@unesp.br (L.M.G.); fp.foresti@unesp.br (F.P.-F.) \\ 2 Department of Structural and Functional Biology, Institute of Biosciences, São Paulo State University, \\ Botucatu, São Paulo 18618-689, Brazil; f.foresti@unesp.br (F.F.); claudio.oliveira@unesp.br (C.O.) \\ 3 Mato Grosso State University, Cuiabá, Mato Grosso 78400-000, Brazil; manolopenitente@gmail.com \\ 4 Department of Genetics, Institute of Biological Sciences and Health, Rural Federal University \\ of Rio de Janeiro, Seropedica, Rio de Janeiro 23897-000, Brazil \\ * Correspondence: ut_ricardo@ufrrj.br \\ + Contributed equally to this work.
}

\section{check for}

updates

Citation: Stornioli, J.H.F.; Goes, C.A.G.; Calegari, R.M.; dos Santos, R.Z.; Giglio, L.M.; Foresti, F.; Oliveira, C.; Penitente, M.; Porto-Foresti, F.; Utsunomia, R. The B Chromosomes of Prochilodus lineatus (Teleostei, Characiformes) Are Highly Enriched in Satellite DNAs. Cells 2021, 10, 1527. https://doi.org/10.3390/

cells10061527

Academic Editor: Darren Griffin

Received: 17 May 2021

Accepted: 11 June 2021

Published: 17 June 2021

Publisher's Note: MDPI stays neutral with regard to jurisdictional claims in published maps and institutional affiliations.

Copyright: (c) 2021 by the authors. Licensee MDPI, Basel, Switzerland. This article is an open access article distributed under the terms and conditions of the Creative Commons Attribution (CC BY) license (https:/ / creativecommons.org/licenses/by/ $4.0 /)$.

\begin{abstract}
B or supernumerary chromosomes are dispensable elements that are widely present in numerous eukaryotes. Due to their non-recombining nature, there is an evident tendency for repetitive DNA accumulation in these elements. Thus, satellite DNA plays an important role in the evolution and diversification of B chromosomes and can provide clues regarding their origin. The characiform Prochilodus lineatus was one of the first discovered fish species bearing B chromosomes, with all populations analyzed so far showing one to nine micro-B chromosomes and exhibiting at least three morphological variants ( $\mathrm{Ba}, \mathrm{Bsm}$, and $\mathrm{Bm}$ ). To date, a single satellite DNA is known to be located on the $\mathrm{B}$ chromosomes of this species, but no information regarding the differentiation of the proposed B-types is available. Here, we characterized the satellitome of P. lineatus and mapped 35 satellite DNAs against the chromosomes of $P$. lineatus, of which six were equally located on all B-types and this indicates a similar genomic content. In addition, we describe, for the first time, an entire population without B chromosomes.
\end{abstract}

Keywords: Prochilodus; Curimbatá; Cytogenomics; repetitive DNA; supernumerary chromosomes; concerted evolution; Teleostei

\section{Introduction}

Repetitive portions of eukaryotic genomes can be classified into dispersed elements, such as transposable elements and tandemly arrayed sequences, which encompass multigene families and satellite DNAs (satDNAs) [1,2]. With the exception of the multigene families, repetitive DNAs constitute a heterogeneous collection of sequences that differ in organization, composition, and location but have no clear functions $[1,3]$.

SatDNAs are monomeric units with variable sizes arranged in a head-to-tail fashion that can span up to millions of repetitions primarily located in, but not restricted to, centromeric and pericentromeric heterochromatin [4-6]. Every species carries a catalog of satDNAs that exhibit a wide diversity of sequence families and usually emerge independently from distinct genomic regions by de novo duplications, occasionally followed by clustering [7]. As the main components of heterochromatin, satDNAs are prone to accumulation in regions of low recombination (or recombination-free), which include sex and B chromosomes due to processes that are analogous to Muller's ratchet [4,5,8-10].

B chromosomes are selfish supernumerary elements that usually do not obey the Mendelian laws of inheritance [9]. They typically arise from the A chromosomes of their 
species by chromosomal breakage and accumulate in a natural population by drive mechanisms (e.g., when transmission rates of chromosomes are higher than 0.5$)[9,11]$. Repetitive DNA plays a key role in the differentiation of $B$ chromosomes (due to their non-recombining nature) and can also provide clues regarding the origin of these elements [12-18].

Prochilodus lineatus is a migratory characiform fish with a diploid number of 54 chromosomes. In addition, all populations of $P$. lineatus analyzed to date exhibit a B chromosome system composed of at least three micro-B variants [14,19-21]. Likewise, congeneric species also show similar micro-B chromosomes and it is hypothesized that these elements originated in the last common ancestor of several Prochilodus species [22,23]. In a pioneering study, Jesus et al. [24] isolated two pericentromeric satDNAs from the genome of P. lineatus (SATH1 and SATH2) out of which one (SATH1) was also clustered on the B chromosomes, suggesting an intraspecific origin for these elements [14,24]. This is one of the most wellstudied B chromosome systems in fish [14,19-21,25-29] and the knowledge on its molecular composition is restricted to a single satDNA.

In recent years, the massive characterization of DNA repeats has become feasible owing to the development of next generation sequencing technologies and associated pipelines [7,30-32]. Here, we sequenced the genome of a P. lineatus specimen carrying B chromosomes and characterized its satellitome by combining computational and cytogenetic tools, with a focus on the accumulation of satDNAs on the B chromosomes of this species.

\section{Materials and Methods}

\subsection{Ethics}

The procedures for sampling, maintenance, and analysis of the animals were carried out in accordance with the international rules on animal experimentation followed by the Ethics Committee on the Use of Animals at the São Paulo State University (IBB/UNESP), protocol 1204-CEUA/2019. Animals were collected in accordance with Brazilian environmental protection legislation (Collection Permission MMA/IBAMA/SISBIO—number 3245).

\subsection{Sampling, Chromosomal Preparations, and Genomic DNA Isolation}

Live specimens of $P$. lineatus were collected in two distinct sampling points. Looking for individuals bearing the three described B-types, we collected nine individuals (F1 from wild-caught $P$. lineatus collected in the Sapucaí river at the Upper Paraná River basin, $20^{\circ} 34^{\prime} 20.669^{\prime \prime} \mathrm{S} 47^{\circ} 46^{\prime} 57.868^{\prime \prime}$ W) in the tanks of a fish farm in Sales de Oliveira, São Paulo, Brazil. We also collected 20 specimens of P. lineatus in the Apa river (Paraguay River basin, $22^{\circ} 14^{\prime} 58.96^{\prime \prime} \mathrm{S} 56^{\circ} 55^{\prime} 36.728^{\prime \prime} \mathrm{W}$ ). Fin clips from all individuals were fixed in $95 \%$ ethanol. Mitotic chromosomes were obtained from kidney cells following the protocol established by Foresti et al. [33] (Sapucaí river) and by Foresti et al. [34] (Apa river). After analysis, specimens were fixed in 10\% formaldehyde, preserved in 70\% ethanol, and deposited in the fish collection of the Fish Genetics Laboratory, Faculty of Sciences, São Paulo State University in Bauru-SP under the voucher numbers LG13393-LG13407 (Sapucaí river) and LG13501-13520 (Apa river).

In order to characterize the satellitome of $P$. lineatus, we selected one specimen carrying three B chromosomes and extracted genomic DNA from fin clips using the Wizard Genomic DNA Purification Kit (Promega) following the manufacturer's instructions. After that, we checked DNA quality with NanoDrop (Thermo Fisher Scientific) and 1\% agarose gel electrophoresis.

\subsection{Genome Sequencing and Bioinformatic Analyses}

We sequenced gDNA from a P. lineatus specimen on the BGISEQ-500 platform (pairedend $2 \times 100 \mathrm{bp}$ ) at BGI (BGI Shenzhen Corporation, Shenzhen, China). Raw reads are available in the sequence read archive (SRA-NCBI) under the accession number SRR11676686. In total, we sequenced $3.64 \mathrm{~Gb}$, resulting in a sequencing coverage of approximately $2.14 \times$, under the assumption that the genome of P. lineatus has an estimated size of $1.7 \mathrm{~Gb}$ [35]. 
After quality and adapter trimming with Trimmomatic [35], we characterized the satDNAs of P. lineatus using several iterations of the TAREAN tool [36] followed by filtering the identified satDNAs with DeconSeq [37] until no further satDNA was found. Thereafter, we performed additional iterations with the RepeatExplorer1 software [30], followed by filtering the discovered satDNAs with DeconSeq until no satDNA was found (satMiner protocol). Next, we filtered and removed other tandemly repeated elements, such as multigene families, and performed a homology search to group the sequences into the same sequence variant, family, and superfamily if their identities were greater than $95 \%$, $80 \%$, or $50 \%$, respectively. We then estimated divergence and abundance values using the RepeatMasker software upon a random selection of $2 \times 5,000,000$ reads [38]. In this analysis, we mapped the selected reads against the whole catalog of satDNAs so that the number of mapped reads divided by the total number of analyzed nucleotides would indicate the relative abundances of each satDNA. At the same time, the divergence values are represented as weighted average Kimura divergence values for each repeat family. The satDNA families were named according to Ruiz-Ruano et al. [7], with each satDNA beginning with a species abbreviation (e.g., Pli for P. lineatus) followed by the term "Sat" and a catalog number in order of decreasing abundance followed by the consensus monomer length. Each satDNA was deposited in GenBank with accession numbers MZ161094MZ161143. Furthermore, consensus sequences for each of the 51 satDNA families were manually analyzed to design PCR primers. If the monomer of a given satDNA was shorter than $30 \mathrm{nt}$, we did not design primers and this resulted in a total of 38 primer pairs to be amplified in PCR reactions.

Since four characiform species already had their satellitomes characterized, we BLASTsearched [39] the satellitome of $P$. lineatus against the NCBI nucleotide collection to investigate whether there were any conserved satDNA families within this order. To perform this, we filtered the results by excluding alignments smaller than the size of the satellite monomers and aligned the consensus sequences using MUSCLE [40].

The above-mentioned analysis identified several satDNAs $(n=8$; PliSat12, PliSat15, PliSat18, PliSat21, PliSat30, PliSat36, PliSat40, and PliSat43) in Megaleporinus macrocephalus (described below) and one (PliSat26) in all the Characiformes species, previously known as CharSat01 [41]. We then downloaded genomic libraries from M. macrocephalus (SRA accession number: SRR7263034) to perform comparative analyses. First, we generated multiple satDNA coverage depths and sequence variant profiles using the RepeatProfiler pipeline [42] to visualize their tandem organization and sequence variation in the referred genomes. In order to perform this, we first randomly selected $2 \times 1,000,000$ reads $(2 \times 101 \mathrm{nt})$ from both species. We then concatenated monomers of the target satDNAs to a minimum of $200 \mathrm{nt}$ and text-provided ten single-copy fish genes to be mapped for single-copy normalization of the read coverage (ppfia1 (XM_022685633.1), foxl2 (XM_007232295.3), prospero (XM_017708821.1), msh4 (XM_017711771.1), zdhhc22 (XM_017711775.1), coq6 (XM_017711829.1), znf106 (XM_017711848.1), lactamase (XM_022682177.1), gastrula zinc finger (XM_022685636.1), and tubulin-kinase (XM_017711762.1)), as described by dos Santos et al. [41]. Read mapping was performed with Bowtie2 [43] with the preset values "-sensitive" and "-no-mixed."

The genomic abundances and Kimura 2-parameter divergence values were estimated independently by sampling $2 \times 1,000,000$ reads $(2 \times 101 \mathrm{nt})$ from both species and aligning them to the eight shared satDNAs. Reads of both species were separately mapped against concatenated monomers of satDNA consensus sequences (spanning 200 nucleotides) using RepeatMasker. Next, we used the calcDivergenceFromAlign.pl script from the RepeatMasker utils and created repeat landscapes so that the number of mapped reads divided by the total of analyzed nucleotides would indicate the relative abundances of each satDNA (represented on the y-axis of the landscape). In contrast, the divergence between the mapped reads and consensus sequences would indicate the Kimura-2-parameter distances (further represented in bins of zero to $70 \%$ ). Thus, copies with low divergence bins are very similar to the consensus sequence. 


\subsection{Fluorescent In Situ Hybridization (FISH)}

Prior to FISH experiments, 35 satDNA probes were labeled with digoxigenin-11-dUTP in PCR reactions. FISH was performed using the method described in Pinkel et al. [44] with small modifications as described in Utsunomia et al. [45]. Briefly, chromosomes were treated with RNAse $(50 \mu \mathrm{g} / \mathrm{mL})$ for $50 \mathrm{~min}$, followed by chromosomal DNA denaturation in $70 \%$ formamide $/ 2 \times \mathrm{SSC}$ for $2 \mathrm{~min}$ at $70{ }^{\circ} \mathrm{C}$. Post-hybridization, slides were washed in $0.2 \times \mathrm{SSC} / 15 \%$ formamide for $20 \mathrm{~min}$ at $42{ }^{\circ} \mathrm{C}$, followed by a second wash in $0.1 \times$ SSC for $15 \mathrm{~min}$ at $60^{\circ} \mathrm{C}$, and a final wash in $4 \times$ SSC $/ 0.5 \%$ Tween for $10 \mathrm{~min}$ at room temperature. Probe detection was performed with anti-digoxigenin-rhodamine (Roche) and the chromosomes were counterstained with DAPI $\left(4^{\prime}, 6\right.$-diamino-2-phenylindole, Vector Laboratories) and analyzed using an optical microscope (Olympus BX61). Images were captured with the DP Controller software (Olympus). A minimum of 10 cells from each individual were analyzed to confirm the observed FISH patterns.

In order to locate multiple probes in the same metaphase plate, especially those mapping simultaneously in A and B chromosomes (e.g., PliSat03, PliSat04, and PliSat05), we performed multiple rounds of FISH with the mentioned probes. In this case, we first performed a regular FISH with a single probe and captured several images. Next, we removed the coverslips and washed the slides with $4 \times$ SSC $/ 1 \%$ Triton- 100 for $5 \mathrm{~min}$. Thereafter, we proceeded with the hybridization protocol again with a reduction of $70 \%$ formamide $/ 2 \times$ SSC and denaturation time to $30 \mathrm{~s}$. Using this approach, we could successfully map the satDNA sequences for five consecutive rounds.

\section{Results}

\subsection{Karyotypes and the First Description of a Population without B Chromosomes}

All the P. lineatus specimens analyzed exhibited a diploid chromosome number of $2 n=54$ and were primarily metacentric and submetacentric chromosomes. In addition, mitotically stable micro-B chromosomes were identified in all individuals collected from the Sapucaí river, as expected (Penitente unpublished data). The B chromosome numbers per cell varied between one and five morphologically distinguishable types, including three variants which are metacentric $(\mathrm{Bm})$, submetacentric $(\mathrm{Bsm})$, and acrocentric $(\mathrm{Ba})$, as described previously (Figure 1) [21]. Remarkably, B chromosomes were not identified in the Apa River population (Figure 1), which is the first report of a P. lineatus population without B chromosomes.
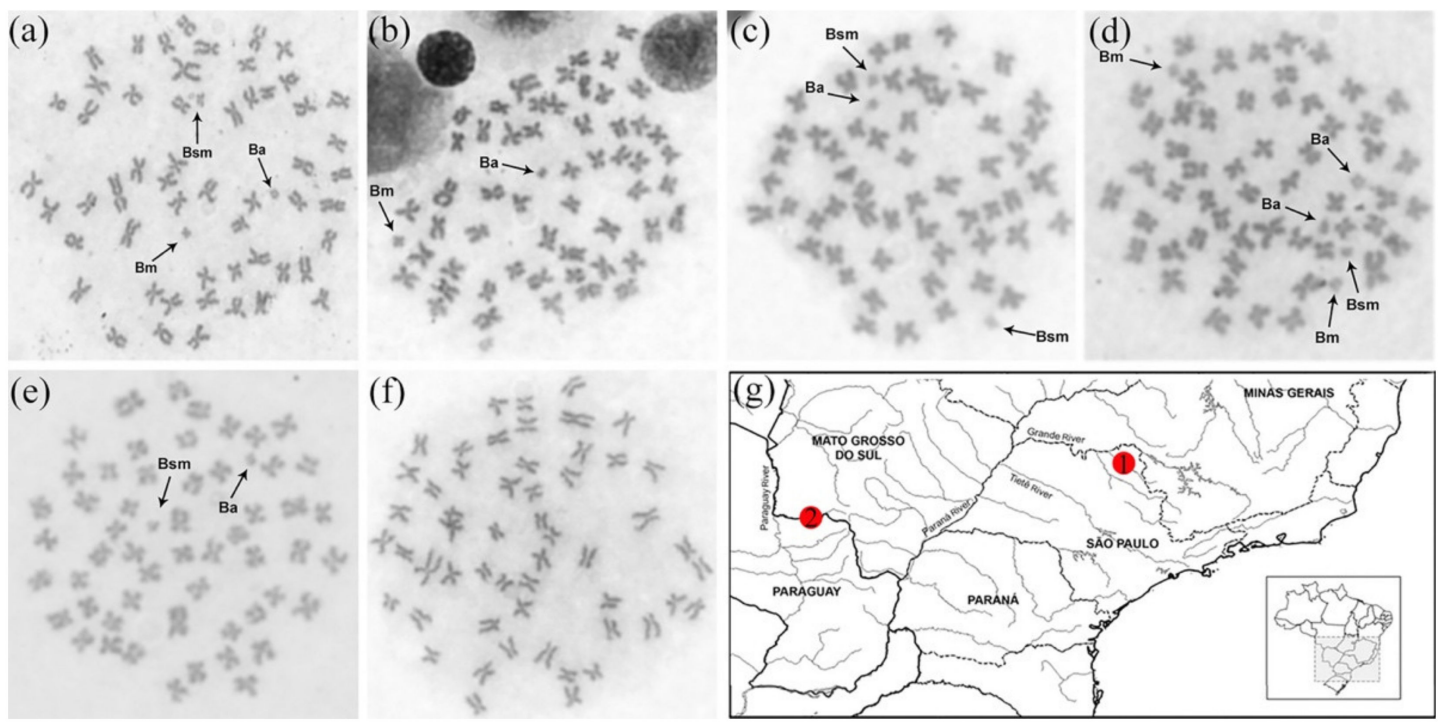

Figure 1. Metaphase plates and collection points. (a-e): metaphase plates from individuals collected in the Sapucaí river and evidences the occurrence of distinct B-types; (f) example of metaphase plate from individuals collected in the Apa river and evidences the absence of B chromosomes; (g) map indicating the sampling points: (1) Sapucaí river; (2) Apa river. 


\subsection{The B chromosomes of P. lineatus Are Highly-Enriched in Satellite DNAs}

After three iterations of TAREAN and one iteration using the RepeatExplorer1 protocol, we found 51 satDNA families for $P$. lineatus, with the repeat unit lengths ranging from 6 to $3008 \mathrm{bp}$, with a median of $59 \mathrm{bp}$ (Table 1). The A + T content of the satDNA families varied from $40 \%$ to $76.2 \%$, with a mean value of $55.24 \%$, and 39 of the satellites showed an $\mathrm{A}+\mathrm{T}$ content greater than $50 \%$. The homology search for the P. lineatus satDNA families revealed two superfamilies (SF1-PliSat01 and PliSat09; SF2-PliSat33 and PliSat42). Among the 51 satDNA families, we designed 38 primer pairs (Table S1) out of which 35 yielded ladder-like patterns after PCR reactions (Table S1) and confirmed their tandem organization. In total, 35 FISH probes were constructed; after the FISH experiments, only eight showed a clustered organization in the P. lineatus chromosomes, whereas the remaining satDNAs were considered non-clustered at the chromosomal level.

Primarily, clustered satDNAs were observed in the centromeric or telomeric regions in addition to those observed exclusively in the B chromosomes (Figure 2). Importantly, we did not notice any difference in the clustering patterns of satDNAs on the distinct $P$. lineatus B-variants. Finally, the satDNA distribution in the Apa river individuals was found to be similar to that observed in individuals from the Sapucaí river, with the exception of PliSat14 which was exclusively mapped on the B chromosomes (Figure 2 and Figure S1).
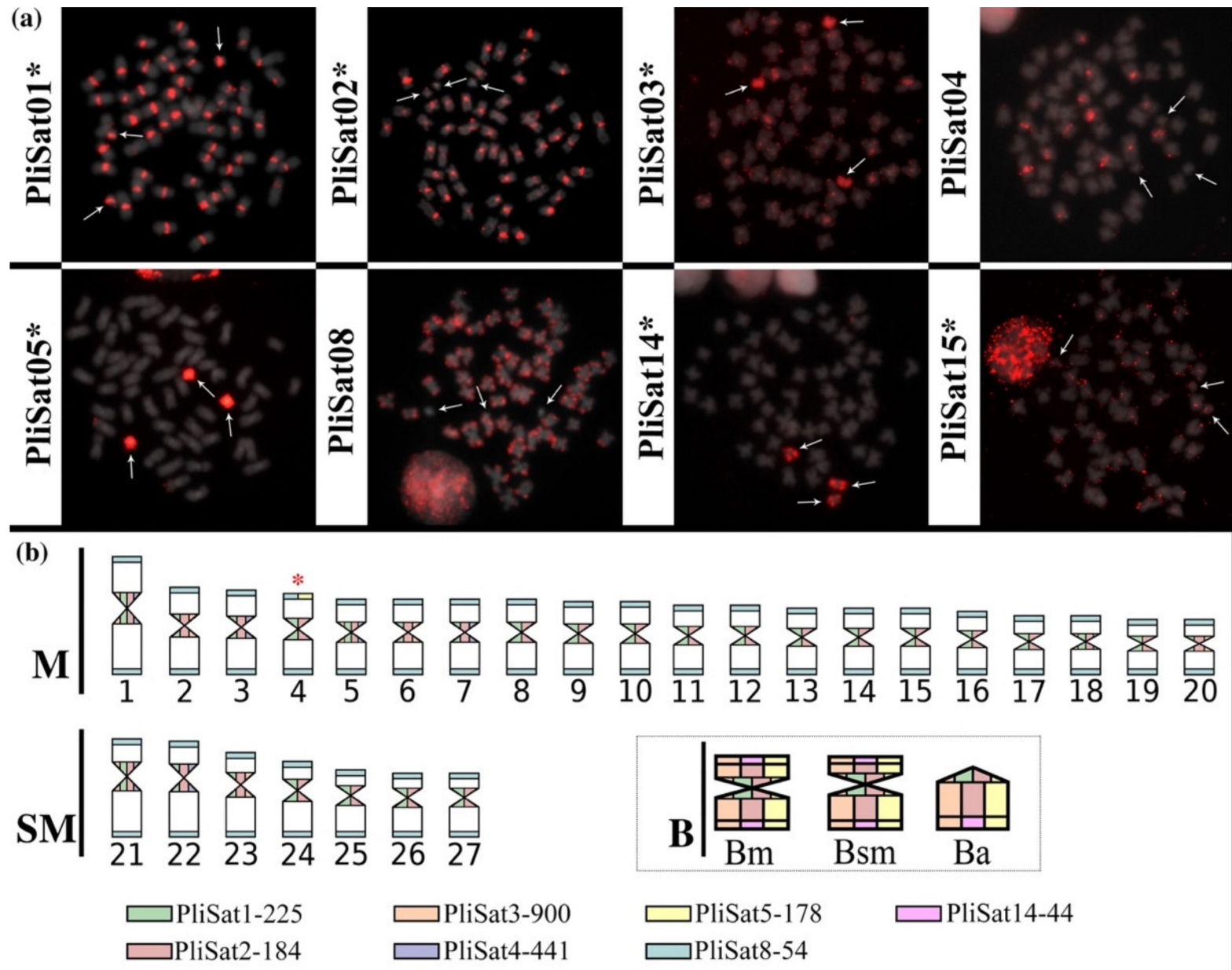

Figure 2. Chromosomal location of satellite DNA sequences. (a) Metaphase plates containing the three described B-types after FISH with different satDNA probes and evidences the similar hybridization patterns for all tested probes. (b) Idiogram of $P$. lineatus karyotype highlighting the distribution of different satDNA families. The B-types shares all the satDNAs (gray box) and are probably derived from pair No. 4 (red asterisk). 
Table 1. Main features of the 51 satDNA families found in P. lineatus genome. Clustered satDNAs are highlighted in gray.

\begin{tabular}{|c|c|c|c|c|c|}
\hline SF & satDNA Family & RUL & $\mathrm{A}+\mathrm{T}(\%)$ & Abundance (\%) & Divergence (\%) \\
\hline \multirow[t]{8}{*}{1} & PliSat01-225 & 225 & 64.5 & $1.332 \times 10^{-2}$ & 7.25 \\
\hline & PliSat02-184 & 184 & 54.9 & $9.882 \times 10^{-3}$ & 10.89 \\
\hline & PliSat03-900 & 900 & 56.5 & $4.829 \times 10^{-3}$ & 1.84 \\
\hline & PliSat04-441 & 441 & 57.9 & $3.191 \times 10^{-3}$ & 6.43 \\
\hline & PliSat05-178 & 178 & 64.6 & $3.097 \times 10^{-3}$ & 8.99 \\
\hline & PliSat06-44 & 44 & 47.8 & $2.021 \times 10^{-3}$ & 13.85 \\
\hline & PliSat07-3008 & 3008 & 63.4 & $1.920 \times 10^{-3}$ & 3.31 \\
\hline & PliSat08-54 & 54 & 62.9 & $1.847 \times 10^{-3}$ & 4.04 \\
\hline \multirow[t]{24}{*}{1} & PliSat09-495 & 495 & 58.0 & $1.809 \times 10^{-3}$ & 5.78 \\
\hline & PliSat10-25 & 25 & 40.0 & $1.215 \times 10^{-3}$ & 11.61 \\
\hline & PliSat11-709 & 709 & 50.4 & $1.120 \times 10^{-3}$ & 9.90 \\
\hline & PliSat12-42 & 42 & 52.4 & $9.841 \times 10^{-4}$ & 13.58 \\
\hline & PliSat13-1928 & 1928 & 62.4 & $8.420 \times 10^{-4}$ & 7.86 \\
\hline & PliSat14-44 & 44 & 50.0 & $5.822 \times 10^{-4}$ & 7.52 \\
\hline & PliSat15-75 & 75 & 61.3 & $4.322 \times 10^{-4}$ & 3.33 \\
\hline & PliSat16-16 & 67 & 58.2 & $3.913 \times 10^{-4}$ & 3.79 \\
\hline & PliSat17-21 & 21 & 76.2 & $3.087 \times 10^{-4}$ & 15.05 \\
\hline & PliSat18-40 & 40 & 57.5 & $2.978 \times 10^{-4}$ & 10.47 \\
\hline & PliSat19-30 & 30 & 56.7 & $2.642 \times 10^{-4}$ & 12.96 \\
\hline & PliSat20-54 & 54 & 57.4 & $2.558 \times 10^{-4}$ & 9.63 \\
\hline & PliSat21-59 & 59 & 49.2 & $2.382 \times 10^{-4}$ & 4.69 \\
\hline & PliSat22-37 & 37 & 45.9 & $2.120 \times 10^{-4}$ & 4.59 \\
\hline & PliSat23-39 & 39 & 53.8 & $1.860 \times 10^{-4}$ & 10.87 \\
\hline & PliSat24-31 & 31 & 51.6 & $1.846 \times 10^{-4}$ & 17.13 \\
\hline & PliSat25-34 & 34 & 47.1 & $1.611 \times 10^{-4}$ & 5.42 \\
\hline & PliSat26-52 & 52 & 59.6 & $1.569 \times 10^{-4}$ & 8.83 \\
\hline & PliSat27-1683 & 1683 & 52.0 & $1.368 \times 10^{-4}$ & 2.25 \\
\hline & PliSat28-32 & 32 & 56.8 & $1.361 \times 10^{-4}$ & 14.45 \\
\hline & PliSat29-60 & 60 & 45.0 & $1.251 \times 10^{-4}$ & 3.31 \\
\hline & PliSat30-31 & 31 & 54.8 & $1.225 \times 10^{-4}$ & 16.69 \\
\hline & PliSat31-707 & 707 & 53.6 & $1.188 \times 10^{-4}$ & 4.27 \\
\hline & PliSat32-30 & 30 & 66.3 & $1.185 \times 10^{-4}$ & 12.19 \\
\hline \multirow[t]{8}{*}{2} & PliSat33-6 & 6 & 57.1 & $1.040 \times 10^{-4}$ & 25.51 \\
\hline & PliSat34-39 & 39 & 53.8 & $1.013 \times 10^{-4}$ & 7.97 \\
\hline & PliSat35-1128 & 1128 & 61.0 & $9.474 \times 10^{-5}$ & 10.43 \\
\hline & PliSat36-68 & 68 & 63.2 & $9.039 \times 10^{-5}$ & 5.55 \\
\hline & PliSat37-554 & 554 & 50.0 & $8.823 \times 10^{-5}$ & 5.47 \\
\hline & PliSat38-32 & 32 & 53.1 & $8.486 \times 10^{-5}$ & 6.29 \\
\hline & PliSat39-915 & 915 & 54.3 & $7.846 \times 10^{-5}$ & 1.35 \\
\hline & PliSat40-27 & 27 & 48.8 & $7.708 \times 10^{-5}$ & 14.00 \\
\hline \multirow[t]{11}{*}{2} & PliSat41-162 & 162 & 45.8 & $7.486 \times 10^{-5}$ & 1.75 \\
\hline & PliSat42-24 & 24 & 55.6 & $6.696 \times 10^{-5}$ & 7.75 \\
\hline & PliSat43-32 & 32 & 53.1 & $6.279 \times 10^{-5}$ & 9.61 \\
\hline & PliSat44-1134 & 1134 & 51.6 & $6.220 \times 10^{-5}$ & 3.43 \\
\hline & PliSat45-340 & 340 & 50.6 & $6.194 \times 10^{-5}$ & 4.83 \\
\hline & PliSat46-29 & 29 & 65.5 & $6.009 \times 10^{-5}$ & 13.97 \\
\hline & PliSat47-574 & 574 & 53.8 & $5.280 \times 10^{-5}$ & 6.89 \\
\hline & PliSat48-387 & 387 & 48.8 & $4.802 \times 10^{-5}$ & 5.00 \\
\hline & PliSat49-67 & 67 & 49.3 & $4.517 \times 10^{-5}$ & 4.30 \\
\hline & PliSat50-48 & 48 & 58.3 & $4.280 \times 10^{-5}$ & 9.07 \\
\hline & PliSat51-911 & 911 & 55.0 & $3.897 \times 10^{-5}$ & 5.00 \\
\hline
\end{tabular}

SF: Superfamily. RUL: repeat unit length. 


\subsection{P. lineatus and M. macrocephalus Share Several satDNAs}

BLAST searches against the NCBI database nucleotide collection revealed multiple significant hits. Among them, we found the following: (i) One 52 nt-long satDNA (PliSat26) was shared among M. macrocephalus, Characidium gomesi, and Astyanax paranae and this sequence was previously known as CharSat01-52 and is conserved within the Characiformes clade [41]; (ii) one satDNA (PliSat8) was shared with Semaprochilodus taeniurus (Figure S2) and this sequence was previously characterized in the $\mathrm{W}$ chromosome of the referred species (known as STW4, accession number: JX157128.1; [46]) and seemed conserved in the family Prochilodontidae; (iii) eight satDNAs (PliSat12, PlisSat15, PliSat18, PliSat21, PliSat30, PliSat36, PliSat40, and PliSat43) were shared with M. macrocephalus (Figure 3, Figure S2), indicating that these tandem repeats originated before the split of Prochilodontidae and Anostomidae, which also includes two other characiform families (Chilodontidae and Curimatidae) with an approximate divergence time of 50-70 million years according to Kolmann et al. [47].

(a)
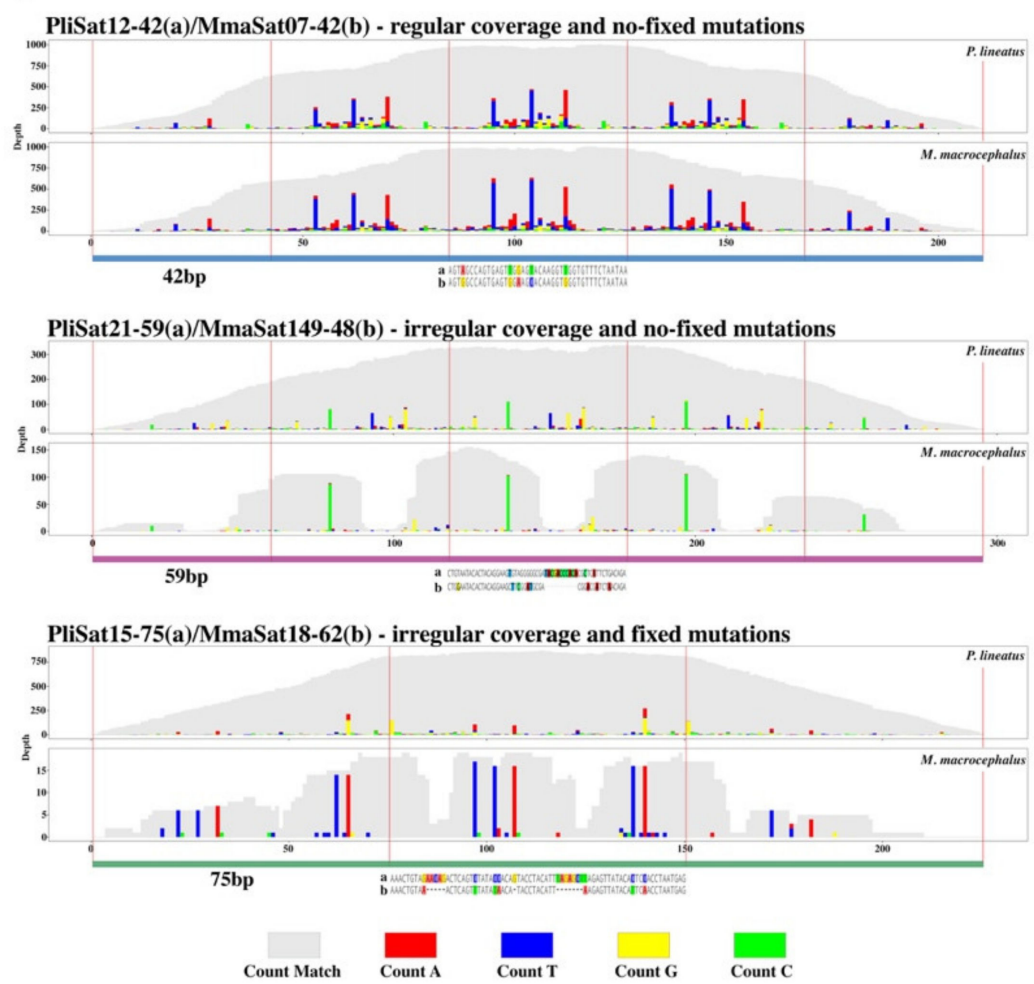

(b)

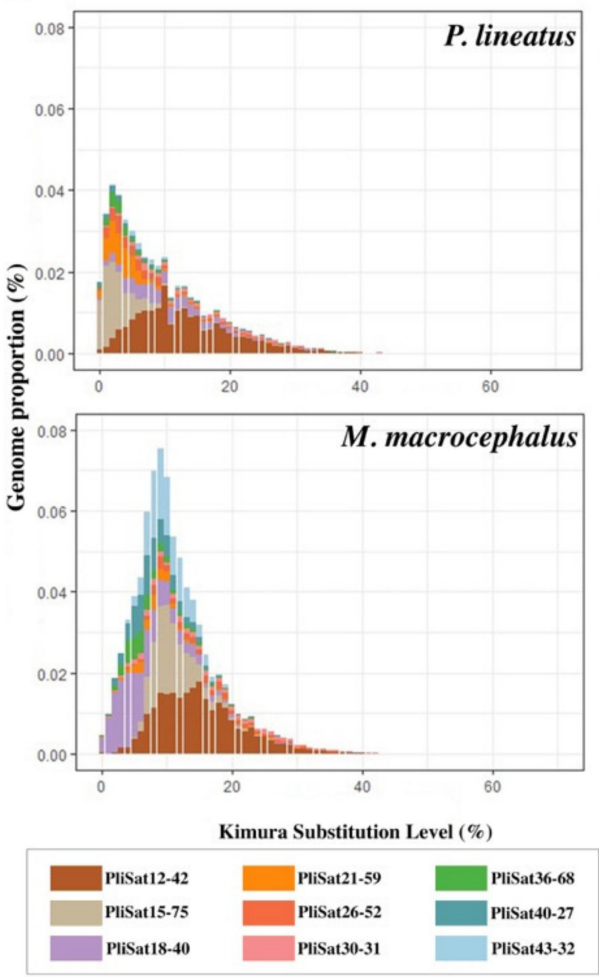

Figure 3. Tandem repeat organization of multiple satellite DNAs in distantly-related species. (a) Examples of variant profiles found for three distinct satDNA families against a consensus sequence. The alignments between consensus satDNA sequences are below the variant profiles (b) Repeat landscapes of the nine shared satellite DNAs detected in the sequenced reads of $P$. lineatus and $M$. macrocephalus. Color-coded bar plots are based on RepeatMasker showing K2P distance to their corresponding consensus sequence on the $\mathrm{x}$ axis and the abundance on the $\mathrm{y}$ axis.

The alignments of eight consensus satDNAs of $P$. lineatus and M. macrocephalus revealed high sequence conservation and the RepeatProfiler and RepeatMasker analyses added new information in this case. Primarily, we observed no consensus sequences of satDNA families showing $100 \%$ identity in the alignments between species (Figure 3). However, the variant profiles provided a genome-wide view of the organization of satDNAs and revealed that six satDNAs (PliSat12, PliSat18, PliSat21, PliSat30, PliSat36, and PliSat40) did not present a fixed mutation (indel or substitution) for a specific species. In this context, even the large gaps observed in the consensus alignments (e.g., PliSat21-59/MmaSat16-51) are not fully fixed (Figure 3a) after millions of years of divergence [47]. Interestingly, 
the variant profiles of PliSat12-42/MmaSat07-42 revealed that this satDNA was highly abundant in both genomes and also exhibited a notable similarity in the SNPs. Finally, the variant profiles of PliSat15-75/MmaSat18-62 and PliSat43-32/MmaSat26-22 showed a higher degree of differentiation with fixed mutations (Figure 3a).

Repeat landscapes were constructed for the above-mentioned conserved satDNAs; these showed that the abundance of sequence variants fluctuates between species, which is evidently noted for PliSat18-40/MmaSat16-51 and PliSat15-75/MmaSat18-62 (Figure 3b). However, we can also note the similar shape of PliSat12-42/MmaSat07-42 in the repeat landscape according to our previous analysis of variant profiles.

\section{Discussion}

\subsection{The Karyotypes of P. lineatus Populations}

The chromosomes of the Prochilodus species have been extensively analyzed in the last 40 years and a conserved karyotype structure with $2 n=54$ chromosomes has been reported to date and is primarily composed of metacentric and submetacentric chromosomes $[23,28,48,49]$. Remarkably, several Prochilodus species exhibit a micro-B chromosome system, which is assumed to have emerged once in this group diverged into multiple B-variants in distinct lineages over time $[21-23,28,50]$. Our results corroborate the common origin of the three observed B-types in P. lineatus, despite it showing morphological differences, all carry the same satDNAs with similar hybridization patterns.

The accumulation of DNA repeats on B chromosomes is a common feature because of their usual non-recombining nature and the predominance of heterochromatic regions $[9,11]$. Considering that the three B-types showed very similar hybridization patterns for all satDNAs and that PliSat05 was highly accumulated on these elements and in a single pair of chromosomes (pair No. 4; Figure S3), we suggest that the B chromosome origin of P. lineatus is related to this chromosome pair. Therefore, a detailed genome-wide analysis of gene content and diversity will permit a better understanding of the B-types, especially because they show different transmission rates [21].

Here, we also describe for the first time an entire population of $P$. lineatus collected from the Paraguay River basin, which does not exhibit B chromosomes. The exclusive and restricted occurrence of $\mathrm{B}$ chromosomes in certain populations of a single species is quite common and has been reported multiple times in many fish species [17,51,52]. In general, this is often attributed to the low vagility of these species, in which populations are geographically isolated and evolve independently [17]. Thus, the case presented herein is noteworthy, since $P$. lineatus is considered one of the most important migratory fish from the Neotropical region [53] and previous analyses indicated that this species has been maintained as a single variable stock in the Paraná system, which includes both of our collection points [54-56]. A hypothesis that could explain the absence of genetic diversity in this species in such a large river basin, such as that of the Paraná River, could be related to the molecular markers (e.g., mitochondrial sequences and microsatellites) in most population genetics studies of this species. Melo et al. [57] revealed little divergence among the mitochondrial lineages in Prochilodus, suggesting that recent episodes of diversification would explain the observed patterns.

The intrapopulation and interpopulation variation of the B-frequency is a common feature and previous studies have shown a correlation between the presence of $B$ chromosomes and environmental conditions, such as temperature, altitude, and rainfall [58-60] as well as seasonal cycles [61]. It is well-known that B chromosomes of multiple species carry active single-copy genes as well as influence A-located gene expression, while satDNAs have been widely described as important gene regulators [41,62-68]. Thus, one hypothesis is that the correlation of $B$ presence with the environment could be due to selective constraints (in favor of or against the presence of B chromosomes). 


\subsection{The Satellitome of Prochilodus lineatus}

Here, we described 51 satDNA families from $P$. lineatus, which is the fifth fish species to have its own characterized satellitome. Remarkably, only eight out of 35 satDNAs were visualized via FISH in P. lineatus, indicating that several satDNAs are organized as short arrays in this genome $(<10 \mathrm{~kb}$, the boundary of FISH sensitivity). Moreover, two satDNA families (PliSat01 and PliSat02) were co-located on all centromeres. However, they did not show any sequence similarity, indicating that two independently originated satDNAs became the centromeric satDNAs in this species including the A and B chromosomes, which might indicate that these satDNAs play a centromeric function.

Regarding the conservation and persistence of satDNAs between distant species, the sharing of nine satDNAs with M. macrocephalus is notable and points to the longterm persistence and conservation of these sequences over millions of years [47]. To date, the long-term evolutionary persistence of satellite DNAs is uncommon and there have been very few described cases [41,67,69-72] primarily because this type of sequence follows a concerted evolutionary pattern that results in high levels of intraspecies sequence homogeneity and low rates of evolutionary persistence $[5,73,74]$. Therefore, we suggest that tandem repeats can naturally persist for millions of years without major changes in their profiles or that this specific maintenance could be related to selective constraints. Importantly, we cannot state that the other 42 satDNAs of P. lineatus were not present in the genomes of other Characiformes species since we only analyzed the consensus sequences of all species outputted from RepeatExplorer and TAREAN pipelines.

\section{Conclusions}

In this study, we characterized the catalog of satDNAs of P. lineatus, which permitted us to confirm the single origin of the B-variants in this species and suggest their origin from a chromosome pair (No. 4) with subsequent morphological diversification. In addition, we found several satDNA families shared between M. macrocephalus, indicating unusual long-term conservation of these sequences. Finally, we described a population of $P$. lineatus that does not exhibit B chromosomes for the first time, which could indicate a certain degree of population differentiation of this species in the Paraná River basin or a possible of correlation between B-presence and environmental conditions.

Supplementary Materials: The following are available online at https://www.mdpi.com/article/ 10.3390/cells10061527/s1, Figure S1: Chromosomal location of satellite DNA sequences in the two analyzed populations, note the similar distribution of satDNA families on the A chromosomes, Figure S2: Tandem repeat organization of multiple satellite DNAs in two distantly related species, examples of variant profiles found for three distinct satDNA families against a consensus sequence, Figure S3: Sequential FISH using multiple probes (PliSat03, PliSat04, and PliSat05), Table S1: Designed primers in the present study.

Author Contributions: Conceptualization, F.P.-F., F.F., and R.U.; Formal analysis, J.H.F.S., C.A.G.G., R.M.C., R.Z.d.S., L.M.G., F.F., C.O., M.P., F.P.-F. and R.U.; Funding acquisition, F.P.-F., F.F., C.O., and R.U.; Supervision, F.P.-F. and R.U.; Validation, J.H.F.S., C.A.G.G., R.M.C., R.Z.d.S., L.M.G., F.F., C.O., M.P., F.P.-F. and R.U.; Writing—original draft, J.H.F.S. and C.A.G.G.; Writing—review and editing, R.U. All authors have read and agreed to the published version of the manuscript.

Funding: This study was supported by grants from CNPq (grant number 405334/2018-4), FAPERJ (grant number 211.475/2019), and FAPESP (grant number 2018/03365-3).

Institutional Review Board Statement: The procedures for sampling, maintenance, and analysis of the animals were carried out in accordance with the international rules on animal experimentation followed by the Ethics Committee on the Use of Animals at the São Paulo State University (IBB/UNESP), protocol 1204-CEUA/2019. Animals were collected in accordance with the Brazilian environmental protection legislation (Collection Permission MMA/ IBAMA/SISBIO—number 3245).

Informed Consent Statement: Not applicable. 
Data Availability Statement: All data reported in this manuscript were deposited on public database. Raw reads were deposited on the sequence read archive (SRA-NCBI, SRR11676686); each satellite DNA consensus sequence was deposited in the GenBank database (MZ161094-MZ161143).

Acknowledgments: We thank D.M.Z.A. Silva, S. Melo, R.F. Artoni, and V.P. Margarido for their valuable suggestions and theoretical contributions; M.B. Cioffi for his valuable suggestions regarding multiple FISH protocols.

Conflicts of Interest: The authors declare no conflict of interest. The funders had no role in the design of the study; in the collection, analyses, or interpretation of data; in the writing of the manuscript or in the decision to publish the results.

\section{References}

1. Charlesworth, B.; Sniegowski, P.; Stephan, W. The evolutionary dynamics of repetitive DNA in eukaryotes. Nature 1994, 371, 215-220. [CrossRef]

2. Jurka, J.; Kapitonov, V.V.; Pavlicek, A.; Klonowski, P.; Kohany, O.; Walichiewicz, J. Repbase Update, a database of eukaryotic repetitive elements. Cytogenet. Genome Res. 2005, 110, 462-467. [CrossRef] [PubMed]

3. Biscotti, M.A.; Olmo, E.; Heslop-Harrison, P. Repetitive DNA in eukaryotic genomes. Chromosom. Res. 2015, 23, 415-420. [CrossRef]

4. Plohl, M.; Meštrović, N.; Mravinac, B. Satellite DNA evolution. Genome Dyn. 2012, 7, 126-152. [PubMed]

5. Garrido-Ramos, M.A. Satellite DNA: An evolving topic. Genes 2017, 8, 230. [CrossRef] [PubMed]

6. Miga, K.H.; Koren, S.; Rhie, A.; Vollger, M.R.; Gershman, A.; Bzikadze, A.; Brooks, S.; Howe, E.; Porubsky, D.; Logsdon, G.A.; et al. Telomere-to-telomere assembly of a complete human X chromosome. Nature 2020, 585, 79-84. [CrossRef] [PubMed]

7. Ruiz-Ruano, F.J.; López-León, M.D.; Cabrero, J.; Camacho, J.P.M. High-throughput analysis of the satellitome illuminates satellite DNA evolution. Sci. Rep. 2016, 6, 1-14. [CrossRef]

8. Green, D.M. Muller's ratchet and the evolution of supernumerary chromosomes. Genome 1990, 33, 818-824. [CrossRef]

9. Camacho, J.P.M.; Sharbel, T.F.; Beukeboom, L.W. B-chromosome evolution. Philos. Trans. R. Soc. B 2000, 355, 163-178. [CrossRef]

10. Charlesworth, D.; Charlesworth, B.; Marais, G. Steps in the evolution of heteromorphic sex chromosomes. Heredity 2005, 95, 118-128. [CrossRef]

11. Houben, A. B chromosomes-A matter of chromosome drive. Front. Plant Sci. 2017, 8, 1-6. [CrossRef]

12. López-León, M.D.; Neves, N.; Schwarzacher, T.; Heslop-Harrison, J.S.; Hewitt, G.M.; Camacho, J.P.M. Possible origin of a B chromosome deduced from its DNA composition using double FISH technique. Chromosom. Res. 1994, 2, 87-92. [CrossRef]

13. Mestriner, C.A.; Galetti, P.M.; Valentini, S.R.; Ruiz, I.R.G.; Abel, L.D.S.; Moreira-Filho, O.; Camacho, J.P.M. Structural and functional evidence that a B chromosome in the characid fish Astyanax scabripinnis is an isochromosome. Heredity 2000, 85, 1-9. [CrossRef]

14. Artoni, R.F.; Vicari, M.R.; Endler, A.L.; Cavallaro, Z.I.; Jesus, C.M.; De Almeida, M.C.; Moreira-Filho, O.; Bertollo, L.A.C. Banding pattern of A and B chromosomes of Prochilodus lineatus (Characiformes, Prochilodontidae), with comments on B chromosomes evolution. Genetica 2006, 127, 277-284. [CrossRef] [PubMed]

15. Teruel, M.; Cabrero, J.; Perfectti, F.; Camacho, J.P.M. B chromosome ancestry revealed by histone genes in the migratory locust. Chromosoma 2010, 119, 217-225. [CrossRef]

16. Silva, D.M.Z.A.; Pansonato-Alves, J.C.; Utsunomia, R.; Araya-Jaime, C.; Ruiz-Ruano, F.J.; Daniel, S.N.; Hashimoto, D.T.; Oliveira, C.; Camacho, J.P.M.; Porto-Foresti, F.; et al. Delimiting the origin of a B chromosome by FISH mapping, chromosome painting and DNA sequence analysis in Astyanax paranae (Teleostei, Characiformes). PLoS ONE 2014, 9, e94896.

17. Utsunomia, R.; Silva, D.M.Z.A.; Ruiz-Ruano, F.J.; Araya-Jaime, C.; Pansonato-Alves, J.C.; Scacchetti, P.C.; Hashimoto, D.T.; Oliveira, C.; Trifonov, V.A.; Porto-Foresti, F.; et al. Uncovering the ancestry of B chromosomes in Moenkhausia sanctaefilomenae (Teleostei, Characidae). PLoS ONE 2016, 11, e0150573. [CrossRef] [PubMed]

18. Serrano-Freitas, E.A.; Silva, D.M.Z.A.; Ruiz-Ruano, F.J.; Utsunomia, R.; Araya-Jaime, C.; Oliveira, C.; Camacho, J.P.M.; Foresti, F. Satellite DNA content of B chromosomes in the characid fish Characidium gomesi supports their origin from sex chromosomes. Mol. Genet. Genom. 2020, 295, 195-207. [CrossRef] [PubMed]

19. Maistro, E.L.; Oliveira, C.; Foresti, F. Cytogenetic analysis of A- and B-chromosomes of Prochilodus lineatus (Teleostei, Prochilodontidae) using different restriction enzyme banding and staining methods. Genetica 2000, 108, 119-125. [CrossRef]

20. Voltolin, T.A.; Laudicina, A.; Senhorini, J.A.; Bortolozzi, J.; Oliveira, C.; Foresti, F.; Porto-Foresti, F. Origin and molecular organization of supernumerary chromosomes of Prochilodus lineatus (Characiformes, Prochilodontidae) obtained by DNA probes. Genetica 2010, 138, 1133-1139. [CrossRef]

21. Penitente, M.; Voltolin, T.A.; Senhorini, J.A.; Bortolozzi, J.; Foresti, F.; Porto-Foresti, F. Transmission rate variation among three B chromosome variants in the fish Prochilodus lineatus (Characiformes, Prochilodontidae). An. Acad. Bras. Cienc. 2013, 85, 1371-1377. [CrossRef]

22. Voltolin, T.A.; Pansonato-Alves, J.C.; Senhorini, J.A.; Foresti, F.; Camacho, J.P.M.; Porto-Foresti, F. Common descent of B Chromosomes in two species of the fish genus Prochilodus (Characiformes, Prochilodontidae). Cytogenet. Genome Res. 2013, 141, 206-211. [CrossRef] 
23. Melo, S.; Utsunomia, R.; Penitente, M.; Sobrinho-Scudeler, P.E.; Porto-Foresti, F.; Oliveira, C.; Foresti, F.; Dergam, J.A. B chromosome dynamics in Prochilodus costatus (Teleostei, Characiformes) and comparisons with supernumerary chromosome system in other Prochilodus species. Comp. Cytogenet. 2017, 11, 393-403. [CrossRef] [PubMed]

24. Jesus, C.M.; Galetti, P.M.; Valentini, S.R.; Moreira-Filho, O. Molecular characterization and chromosomal localization of two families of satellite DNA in Prochilodus lineatus (Pisces, Prochilodontidae), a species with B chromosomes. Genetica 2003, 118, 25-32. [CrossRef]

25. Oliveira, C.; Saboya, S.M.R.; Foresti, F.; Senhorini, J.A.; Bernardino, G. Increased B chromosome frequency and absence of drive in the fish Prochilodus lineatus. Heredity 1997, 79, 473-476. [CrossRef]

26. Dias, A.L.; Foresti, F.; Oliveira, C. Synapsis in supernumerary chromosomes of Prochilodus lineatus (Teleostei: Prochilodontidae). Caryologia 1998, 51, 105-113. [CrossRef]

27. Cavallaro, Z.I.; Bertollo, L.A.C.; Perfectti, F.; Camacho, J.P.M. Frequency increase and mitotic stabilization of a B chromosome in the fish Prochilodus lineatus. Chromosom. Res. 2000, 8, 627-634. [CrossRef] [PubMed]

28. Voltolin, T.A.; Senhorini, J.A.; Oliveira, C.; Foresti, F.; Bortolozzi, J.; Porto-Foresti, F. B-chromosome frequency stability in Prochilodus lineatus (Characiformes, Prochilodontidae). Genetica 2010, 138, 281-284. [CrossRef] [PubMed]

29. Penitente, M.; Daniel, S.N.; Scudeler, P.E.S.; Foresti, F.; Porto-Foresti, F. B chromosome variants in Prochilodus lineatus (Characiformes, Prochilodontidae) analyzed by microdissection and chromosome painting techniques. Caryologia 2016, 69, 181-186. [CrossRef]

30. Novák, P.; Neumann, P.; Pech, J.; Steinhaisl, J.; Macas, J. RepeatExplorer: A Galaxy-based web server for genome-wide characterization of eukaryotic repetitive elements from next-generation sequence reads. Bioinformatics 2013, 29, 792-793. [CrossRef]

31. Lower, S.S.; McGurk, M.P.; Clark, A.G.; Barbash, D.A. Satellite DNA evolution: Old ideas, new approaches. Curr. Opin. Genet. Dev. 2018, 49, 70-78. [CrossRef] [PubMed]

32. Harris, R.S.; Cechova, M.; Makova, K.D. Noise-cancelling repeat finder: Uncovering tandem repeats in error-prone long-read sequencing data. Bioinformatics 2019, 35, 4809-4811. [CrossRef] [PubMed]

33. Foresti, F.; Almeida-Toledo, L.F.; Toledo-Filho, S.A. Polymorphic nature of nucleolus organizer regions in fishes. Cytogenet. Cell Genet. 1981, 31, 137-144. [CrossRef]

34. Fenocchio, A.S.; Bertollo, L.A.C. A simple method for fresh-weter fish lymphocyte culture. Rev. Bras. Genet 1988, 11, 847-852.

35. Bolger, A.M.; Lohse, M.; Usadel, B. Trimmomatic: A flexible trimmer for Illumina sequence data. Bioinformatics 2014, 30, 2114-2120. [CrossRef] [PubMed]

36. Novák, P.; Robledillo, L.Á.; Koblížková, A.; Vrbová, I.; Neumann, P.; Macas, J. TAREAN: A computational tool for identification and characterization of satellite DNA from unassembled short reads. Nucleic Acids Res. 2017, 45, e111. [CrossRef]

37. Schmieder, R.; Edwards, R. Quality control and preprocessing of metagenomic datasets. Bioinformatics 2011, $27,863-864$. [CrossRef]

38. Smit, A.; Hubley, R.; Green, P. RepeatMasker Open-4.0. Available online: http://www.repeatmasker.org (accessed on 22 November 2020).

39. Altschul, S.F.; Gish, W.; Miller, W.; Myers, E.W.; Lipman, D.J. Basic local alignment search tool. J. Mol. Biol. 1990, 215 , 403-410. [CrossRef]

40. Edgar, R.C. MUSCLE: Multiple sequence alignment with high accuracy and high throughput. Nucleic Acids Res. 2004, 32, 1792-1797. [CrossRef]

41. Dos Santos, R.Z.; Calegari, R.M.; Silva, D.M.Z.A.; Ruiz-Ruano, F.J.; Melo, S.; Oliveira, C.; Foresti, F.; Uliano-Silva, M.; Foresti, F.P.; Utsunomia, R. A long-term conserved satellite DNA that remains unexpanded in several genomes of Characiformes fish is actively transcribed. Genome Biol. Evol. 2021, 13, evab002. [CrossRef]

42. Negm, S.; Greenberg, A.; Larracuente, A.M.; Sproul, J.S. RepeatProfiler: A pipeline for visualization and comparative analysis of repetitive DNA profiles. Mol. Ecol. Resour. 2021, 21, 969-981. [CrossRef]

43. Langmead, B.; Salzberg, S.L. Fast gapped-read alignment with Bowtie 2. Nat. Methods 2012, 9, 357-359. [CrossRef]

44. Pinkel, D.; Straume, T.; Gray, J.W. Cytogenetic analysis using quantitative, high-sensitivity, fluorescence hybridization. Proc. Natl. Acad. Sci. USA 1986, 83, 2934-2938. [CrossRef] [PubMed]

45. Utsunomia, R.; Ruiz-Ruano, F.J.; Silva, D.M.Z.A.; Serrano, E.A.; Rosa, I.F.; Scudeler, P.E.S.; Hashimoto, D.T.; Oliveira, C.; Camacho, J.P.M.; Foresti, F. A glimpse into the satellite DNA library in Characidae fish (Teleostei, Characiformes). Front. Genet. 2017, 8, 1-11. [CrossRef] [PubMed]

46. Terencio, M.L.; Schneider, C.H.; Gross, M.C.; Nogaroto, V.; Almeida, M.C.; Artoni, R.F.; Vicari, M.R.; Feldberg, E. Repetitive sequences associated with differentiation of W chromosome in Semaprochilodus taeniurus. Genetica 2012, 140, 505-512. [CrossRef] [PubMed]

47. Kolmann, M.A.; Hughes, L.C.; Hernandez, L.P.; Arcila, D.; Betancur-R, R.; Sabaj, M.H.; López-Fernández, H.; Ortí, G. Phylogenomics of Piranhas and Pacus (Serrasalmidae) Uncovers How Dietary Convergence and Parallelism Obfuscate Traditional Morphological Taxonomy. Syst. Biol. 2020, 70, 576-592. [CrossRef]

48. Pauls, E.; Bertollo, L.A.C. Evidence for a system of supernumerary chromosomes in Prochilodus scrofa Steindachner, 1881 (Pisces, Prochilodontidae). Caryologia 1983, 36, 307-314. [CrossRef]

49. Voltolin, T.A.; Penitente, M.; Mendonça, B.B.; Senhorini, J.A.; Foresti, F.; Porto-Foresti, F. Karyotypic conservatism in five species of Prochilodus (Characiformes, Prochilodontidae) disclosed by cytogenetic markers. Genet. Mol. Biol. 2013, 36, 347-352. [CrossRef] 
50. Pauls, E.; Bertollo, L.A.C. Distribution of a supernumerary chromosome system and aspects of karyotypic evolution in the genus Prochilodus (Pisces, Prochilodontidae). Genetica 1990, 81, 117-123. [CrossRef]

51. Moreira-Filho, O.; Galetti, P.M.; Bertollo, L.A.C. B chromosomes in the fish Astyanax scabripinnis (Characidae, Tetragonopterinae): An overview in natural populations. Cytogenet. Genome Res. 2004, 106, 230-234. [CrossRef]

52. Scacchetti, P.C.; Utsunomia, R.; Pansonato-Alves, J.C.; Vicari, M.R.; Artoni, R.F.; Oliveira, C.; Foresti, F. Chromosomal mapping of repetitive DNAs in Characidium (Teleostei, Characiformes): Genomic organization and diversification of ZW sex chromosomes. Cytogenet. Genome Res. 2015, 146, 136-143. [CrossRef] [PubMed]

53. Speranza, E.D.; Colombo, J.C. Biochemical composition of a dominant detritivorous fish Prochilodus lineatus along pollution gradients in the Paraná-Río de la Plata Basin. J. Fish Biol. 2009, 74, 1226-1244. [CrossRef]

54. Sivasundar, A.; Bermingham, E.; Ortí, G. Population structure and biogeography of migratory freshwater fishes (Prochilodus: Characiformes) in major South American rivers. Mol. Ecol. 2001, 10, 407-417. [CrossRef] [PubMed]

55. Garcez, R.; Calcagnotto, D.; Almeida-Toledo, L.F. Population structure of the migratory fish Prochilodus lineatus (Characiformes) from rio Grande basin (Brazil), an area fragmented by dams. Aquat. Conserv. Mar. Freshw. Ecosyst. 2011, 21, 268-275. [CrossRef]

56. Ferreira, D.G.; Souza-Shibatta, L.; Shibatta, O.A.; Sofia, S.H.; Carlsson, J.; Dias, J.H.P.; Makrakis, S.; Makrakis, M.C. Genetic structure and diversity of migratory freshwater fish in a fragmented Neotropical river system. Rev. Fish Biol. Fish. 2017, 27, 209-231. [CrossRef]

57. Melo, B.F.; Dorini, B.F.; Foresti, F.; Oliveira, C. Little divergence among mitochondrial lineages of Prochilodus (Teleostei, Characiformes). Front. Genet. 2018, 9, 1-9. [CrossRef]

58. Hewitt, G.; Ruscoe, C. Changes in microclimate correlated with a cline for B-chromosomes in the grasshopper Myrmeleotettix maculatus (Thunb.) (Orthoptera: Acrididae). J. Anim. Ecol. 1971, 40, 753. [CrossRef]

59. Néo, D.M.; Moreira-Filho, O.; Camacho, J.P.M. Altitudinal variation for B chromosome frequency in the characid fish Astyanax scabripinnis. Heredity 2000, 85, 136-141. [CrossRef]

60. Vujosevic, M.; Blagojevic, J. Does environment affect polymorphism of B chromosomes in the yellow-necked mouse Apodemus flavicollis? Z. Saugetierkd. 2000, 65, 313-317.

61. Vujošević, M.; Blagojević, J. Seasonal changes of B-chromosome frequencies within the population of Apodemus flavicollis (Rodentia) on Cer mountain in Yugoslavia. Acta Theriol. 1995, 40, 131-137. [CrossRef]

62. Martis, M.M.; Klemme, S.; Banaei-Moghaddam, A.M.; Blattner, F.R.; Macas, J.; Schmutzer, T.; Scholz, U.; Gundlach, H.; Wicker, T.; Sǐmková, H.; et al. Selfish supernumerary chromosome reveals its origin as a mosaic of host genome and organellar sequences. Proc. Natl. Acad. Sci. USA 2012, 109, 13343-13346. [CrossRef] [PubMed]

63. Valente, G.T.; Conte, M.A.; Fantinatti, B.E.A.; Cabral-de-Mello, D.C.; Carvalho, R.F.; Vicari, M.R.; Kocher, T.D.; Martins, C. Origin and evolution of B chromosomes in the cichlid fish Astatotilapia latifasciata based on integrated genomic analyses. Mol. Biol. Evol. 2014, 31, 2061-2072. [CrossRef]

64. Ferreira, D.; Meles, S.; Escudeiro, A.; Mendes-da-Silva, A.; Adega, F.; Chaves, R. Satellite non-coding RNAs: The emerging players in cells, cellular pathways and cancer. Chromosom. Res. 2015, 23, 479-493. [CrossRef]

65. Navarro-Domínguez, B.; Ruiz-Ruano, F.J.; Cabrero, J.; Corral, J.M.; López-León, M.D.; Sharbel, T.F.; Camacho, J.P.M. Proteincoding genes in B chromosomes of the grasshopper Eyprepocnemis plorans. Sci. Rep. 2017, 7, 1-12. [CrossRef] [PubMed]

66. Castro, J.P.; Hattori, R.S.; Yoshinaga, T.T.; Silva, D.M.Z.A.; Foresti, F.; Santos, M.H.; Almeida, M.C.; Artoni, R.F. Differential expression of dmrt1 in Astyanax scabripinnis (Teleostei, Characidade) is correlated with B chromosome occurrence. Zebrafish 2019, 16, 182-188. [CrossRef]

67. Halbach, R.; Miesen, P.; Joosten, J.; Taşköprü, E.; Rondeel, I.; Pennings, B.; Vogels, C.B.F.; Merkling, S.H.; Koenraadt, C.J.; Lambrechts, L.; et al. A satellite repeat-derived piRNA controls embryonic development of Aedes. Nature 2020, 580, $274-277$. [CrossRef] [PubMed]

68. Louzada, S.; Lopes, M.; Ferreira, D.; Adega, F.; Escudeiro, A.; Gama-Carvalho, M.; Chaves, R. Decoding the role of satellite DNA in genome architecture and plasticity-An evolutionary and clinical affair. Genes 2020, 11, 72. [CrossRef] [PubMed]

69. Robles, F.; De La Herrán, R.; Ludwig, A.; Ruiz Rejón, C.; Ruiz Rejón, M.; Garrido-Ramos, M.A. Evolution of ancient satellite DNAs in sturgeon genomes. Gene 2004, 338, 133-142. [CrossRef]

70. Plohl, M.; Petrović, V.; Luchetti, A.; Ricci, A.; Šatović, E.; Passamonti, M.; Mantovani, B. Long-term conservation vs high sequence divergence: The case of an extraordinarily old satellite DNA in bivalve mollusks. Heredity 2010, 104, 543-551. [CrossRef]

71. Chaves, R.; Ferreira, D.; Mendes-Da-Silva, A.; Meles, S.; Adega, F. FA-SAT is an old satellite DNA frozen in several bilateria genomes. Genome Biol. Evol. 2017, 9, 3073-3087. [CrossRef]

72. Lorite, P.; Muñoz-López, M.; Carrillo, J.A.; Sanllorente, O.; Vela, J.; Mora, P.; Tinaut, A.; Torres, M.I.; Palomeque, T. Concerted evolution, a slow process for ant satellite DNA: Study of the satellite DNA in the Aphaenogaster genus (Hymenoptera, Formicidae). Org. Divers. Evol. 2017, 17, 595-606. [CrossRef]

73. Dover, G. Molecular drive: A cohesive mode of species evolution. Nature 1982, 299, 111-117. [CrossRef] [PubMed]

74. Garrido-Ramos, M.A. Satellite DNA in plants: More than just Rubbish. Cytogenet. Genome Res. 2015, 146, 153-170. [CrossRef] [PubMed] 